\title{
Research and Publication from Developing Countries
}

\author{
Mohan Raj Sharma, MBBS, MS \\ Professor and Head of Neurosurgery, Maharajgunj Medical Campus, TU Teaching Hospital \\ Executive Editor, Journal of Institute of Medicine Nepal \\ Email:mohanrajsharma@gmail.com
}

\section{INTRODUCTION}

"n n 2002, Richard Smith wrote an editorial, "publishing research from developing countries" in the Journal "Statistics in Medicine" highlighting the importance of research and publication from the developing countries (DCs). In that article, he mentioned the disparity in research and publication between the developed and developing countries. Almost two decades on, the problem still largely remains the same.

It is estimated that more than $80 \%$ of the world's population lives in more than 100 developing countries. $^{2}$ In terms of disease burden, the prevalence and mortality from diseases in the low and middle-income countries are disproportionately high compared to developed countries. ${ }^{3}$ Although there is a high burden of disease, we base our treatment inferring results from research and publication from the developed countries which may not be fully generalizable due to geographical cultural, racial, and economic factors. This is where the problem lies.

Although globalization has significantly bridged the gap in many areas (film and music industries, fashion, telecommunication, etc.), unfortunately, it is not so when it comes to health research. Apart from a few collaborative research, DCs have contributed very little to the world body of knowledge. Very few major studies that change the treatment have ever been carried out from South Asia. We have to take the results from the research done on the western population at face value. The global concept in treatment and education is gaining momentum recently. The National Surgical, Obstetric, and Anesthesia Plan (NSOAP) has been developed globally as a policy to address the high burden of surgical diseases in the DCs. However, no such concerted effort seems in place for research.

Good access to scientific materials is a prerequisite to research. ${ }^{4}$ Scientists and scholars in DCs now have access to the articles from world-class journals to keep them up-to-date, thanks to many open access journals now available worldwide. However, this comes with a price. Open access journals from the developed countries are hurting the scientists simultaneously as they charge a lot for publication to offset the cost of making publications open access. Therefore, the little research that is carried out in low-income settings is not noticed on the world stage because of this cost barrier to publication. As a side effect of this, the researchers from DCs are the favorite targets of predatory journals that publish the articles with no or little peer review.

It is high time that we develop research and publication culture in our institutions. The tradition of doing research and publication only to meet the eligibility criteria to sit for the exam or promotion should be broken. Better incentives for research and publication locally, nationally and regionally will help encourage the young researchers in this regard. There is a huge scope of research on many disease conditions endemic to us. We have ideal settings for teaching and training because of the large volume and variety of cases. But there is a huge gap between what we have done and how much we have published our work. Nepalese scientists and clinicians should come forward to fill this chasm.

\section{REFERENCES}

1. Smith R. Publishing research from developing countries. Statist med. 2002;22:2869-2877.

2. Sharma MR, Marsh $H$. Challenges in global neurosurgery. In: Ellenbogen RG, Sekhar LN, Kitchen N (Eds). Principles of Neurological Surgery. Fourth edition. New York: Elsevier; 2018. pp 8-14.

3. NCD Countdown 2030 collaborators. NCD Countdown 2030: worldwide trends in non-communicable disease mortality and progress towards Sustainable Development Goal target 3.4. Lancet. 2018; 22;392(10152):1072-1088.

4. Dekeyser R. Scientific research and information in developing countries: Necessity, threats, and opportunities. 2012. https://pdfs.semanticscholar.org/ b962/61e71e186bc933235a65f2c38b73349981db.pdf. Accessed November 12, 2020 